\title{
AUTOMORPHISMS OF KRONROD-REEB GRAPHS OF MORSE FUNCTIONS ON 2-TORUS
}

\author{
ANNA KRAVCHENKO AND BOHDAN FESHCHENKO
}

\begin{abstract}
This paper is devoted to a study of special subgroups of automorphism groups of Kronrod-Reeb graphs of Morse functions on 2-torus $T^{2}$ which arise from actions of diffeomorphisms preserving a given Morse function on $T^{2}$. In this paper we give a full description of such classes of groups.
\end{abstract}

\section{INTRODUCTION}

Topological graphs naturally arise from a study of smooth functions on smooth manifolds as powerful tools which contain "combinatorial" information about a given smooth function and hence the information about the topology of the smooth manifolds. KronrodReeb graphs of Morse functions on compact manifolds, named after G. Reeb by R. Thom and after A. Kronrod by V. Sharko are famous examples of such graphs. They were studied by many authors. E.g. the problem of realization of a graph as a Kronrod-Reeb graph of a Morse (in general smooth) function on a given smooth compact manifold was proposed by V. Sharko and were studied in the papers of V. Sharko [31], Y. Masumoto and O. Saeki [24], Ł. Michalak [26], K. Cole-McLaughlin et al [1], M. Kaluba, W. Marzantowicz, N. Silva [7] and others. This problem is closely related to another important problem of topological conjugacy for Morse functions on smooth manifolds, see e.g. E. Kulinich [14], V. Sharko [30], D. Lychak and O. Prishlyak [15]. E. Polulyakh in [27, 28] has generalized the notion of the Kronrod-Reeb "graph" for functions on non-compact surfaces.

Homotopy properties of Morse functions on smooth surfaces were studied by V. Sharko [29], H. Zieschang, S. Matveev, E. Kudryavtseva [11], K. Ikegami and O. Saeki [5], B. Kalmar [6], S. Maksymenko. We give a short overview of the results of S. Maksymenko $[17,18,19,20,21]$ and E. Kudryavtseva devoted to a study of homotopy properties of orbits and stabilizers of smooth functions on compact surfaces under the action of their diffeomorphism groups. We will see that special subgroups of the automorphism groups of Kronrod-Reeb graphs play an essential role in the description of homotopy types of these spaces.

Let $M$ be a smooth compact surface and $X$ be a closed (possibly empty) subset of $M$. The diffeomorphisms group $\mathcal{D}(M, X)$ acts on the space of smooth functions $C^{\infty}(M)$ by the rule: $C^{\infty}(M) \times \mathcal{D}(M, X) \rightarrow C^{\infty}(M),(f, h) \mapsto f \circ h$. Under this action we denote by

$$
\mathcal{S}(f, X)=\{h \in \mathcal{D}(M, X) \mid f \circ h=f\}, \quad \mathcal{O}(f, X)=\{f \circ h \mid h \in \mathcal{D}(M, X)\}
$$

the stabilizer and the orbit of $f \in C^{\infty}(M)$. Endow $C^{\infty}(M)$ and $\mathcal{D}(M, X)$ with strong Whitney topologies; for a fixed $f \in C^{\infty}(M)$ these topologies induce some topologies on $\mathcal{S}(f, X)$ and $\mathcal{O}(f, X)$. By $\mathcal{D}_{\text {id }}(M, X), \mathcal{S}_{\text {id }}(f, X)$, and $\mathcal{O}_{f}(f, X)$ we denote connected components of the identity map id ${ }_{M}$ of $\mathcal{D}(M, X), \mathcal{S}(f, X)$, and the component of $\mathcal{O}(f, X)$

2020 Mathematics Subject Classification. 57S05, 57R45, 37C05.

Key words and phrases. Automorphisms, graphs, Morse functions. 
which contains $f$, respectively. If $X=\varnothing$ we put $\mathcal{D}(M):=\mathcal{D}(M, \varnothing), \mathcal{S}(f):=\mathcal{S}(f, \varnothing)$, $\mathcal{O}(f):=\mathcal{O}(f, \varnothing)$, and so on.

In this paper we will consider Kronrod-Reeb graphs of Morse functions on smooth compact surfaces. Since these surfaces may have boundary, we need to specify what will be meant by a Morse function. By a Morse function $f$ on a surface $M$ we will mean a smooth function $f \in C^{\infty}(M)$ on a surface $M$ such that $f$ takes constant values on the connected components of the boundary $\partial M$ and each critical point of $f$ is non-degenerate and is contained in $\operatorname{Int}(M)$. A Morse function $f$ on $M$ is called simple if every critical connected component of every critical level set contains a unique critical point, and $f$ is called generic if each level-set of $f$ contains no more than one critical point.

S. Maksymenko, $[17,18,19,20,21]$, showed that if $f$ has at least one saddle point, then $\pi_{n} \mathcal{O}_{f}(f)=\pi_{n} M$ for $n \geq 3, \pi_{2} \mathcal{O}_{f}(f)=0$, and for $\pi_{1} \mathcal{O}_{f}(f)$ there is a short exact sequence

$$
1 \longrightarrow \pi_{1} \mathcal{D}_{\mathrm{id}}(M) \oplus \mathbb{Z}^{k} \longrightarrow \pi_{1} \mathcal{O}_{f}(f) \longrightarrow G(f) \longrightarrow 1
$$

for some $k \geq 0$ and a finite group $G(f)$ which is a group of automorphisms of a KronrodReeb graph of $f$ induced by isotopic to $\operatorname{id}_{M}$ and $f$-preserving diffeomorphisms, i.e., diffeomorphisms from $\mathcal{S}^{\prime}(f)=\mathcal{S}(f) \cap \mathcal{D}_{\text {id }}(M)$. Moreover if $f$ is generic, then the group $G(f)$ is trivial, and $\mathcal{O}_{f}(f)$ is homotopy equivalent to $\left(S^{1}\right)^{m}$ if $M \neq S^{2}$ and $M \neq \mathbb{R} P^{2}$, to $S^{2}$ if $M=S^{2}$ and $f$ have only two critical points, and to $\mathrm{SO}(3) \times\left(S^{1}\right)^{m}$ otherwise, for some $m \geq 0$ depending on $f$. E. Kudryavtseva $[12,13]$ calculated the homotopy types of connected components of the space of Morse functions on compact surfaces, and extend the result about homotopy type of $\mathcal{O}_{f}(f)$ when $G(f)$ is non-trivial. She showed that the space $\mathcal{O}_{f}(f)$ is homotopy equivalent to the following quotient-spaces $\left(S^{1}\right)^{m} / G(f)$ if $M \neq S^{2}$, and to $\mathrm{SO}(3) \times\left(S^{1}\right)^{m} / G(f)$ otherwise, where $G(f)$ freely acts on $\left(S^{1}\right)^{m}$, and the number $m$ is the rank of the Abelian group $\pi_{1} \mathcal{D}_{\text {id }}(M) \oplus \mathbb{Z}^{k}$, see (1). Note that $G(f)$ is the holonomy group of the compact manifold $\left(S^{1}\right)^{m} / G(f)$.

An algebraic structure of $\pi_{1} \mathcal{O}_{f}(f)$ for the Morse functions on 2-torus was described in the series of papers by S. Maksymenko and the second author $[23,16,22,4]$. This is one of non-trivial cases since $\mathcal{D}_{\text {id }}\left(T^{2}\right)$ is not contractible, so the image $\pi_{1} \mathcal{D}_{\text {id }}\left(T^{2}\right)$ in $\pi_{1} \mathcal{O}_{f}(f)$ has non-trivial impact on the algebraic structure of $\pi_{1} \mathcal{O}_{f}(f)$, see $(1)$.

Since groups $G(f)$ play the essential role in the description of homotopy types of $\mathcal{O}_{f}(f)$ S. Maksymenko and A. Kravchenko [10] studied classes of such groups and their subsets. Let $\mathscr{G}(M)$ be the minimal set of isomorphisms classes of groups $G(f)$ for all Morse functions $f$ on $M$. By $\mathscr{G}^{\operatorname{smp}}(M)$ and $\mathscr{G}^{\text {gen }}(M)$ will be denoted subclasses of $\mathscr{G}(M)$ which correspond to classes of isomorphisms of groups $G(f)$ for all simple and generic Morse functions on $M$ respectively. The first author and S. Maksymenko gave a full algebraic description of classes $\mathscr{G}(M)$ and $\mathscr{G}$ smp $(M)$ for all compact oriented surfaces $M$ which are distinct from 2-sphere $S^{2}$ and 2-torus $T^{2}$, and proved that the class $\mathscr{G}^{\mathrm{gen}}(M)$ is trivial, i.e., contains only trivial group $\{1\}$ for all compact oriented surfaces $M$, see Theorem 2.2, or [10]. We also mention that an algebraic structure of $G(f)$ for Morse function on $S^{2}$ is partially understood [8], and in general this case is more complicated than the case of Morse functions on compact surfaces of genus $\geq 1$. Recently S. Maksymenko and A. Kravchenko [9] described special subgroups of $G(f)$ for Morse functions on $S^{2}$.

The aim of this paper is to extend the result of [10] by giving a full description of the classes $\mathscr{G}\left(T^{2}\right)$ and $\mathscr{G}^{s m p}\left(T^{2}\right)$ for a Morse functions on 2-torus.

1.1. Conventions and notations. To state our main result we need the notion of wreath products of groups with cyclic groups. So we recall these definitions. Let $G$ be a group, $m, n \geq 1$ be integers. Consider two effective actions $\alpha: G^{n} \times \mathbb{Z}_{n} \rightarrow G^{n}$, and 
$\beta: G^{n m} \times\left(\mathbb{Z}_{n} \times \mathbb{Z}_{m}\right) \rightarrow G^{n m}$ of $\mathbb{Z}_{n}$ and $\mathbb{Z}_{n} \times \mathbb{Z}_{m n}$ on $G^{n}$ and $G^{n m}$ given by the formulas:

$$
\alpha\left(\left(g_{i}\right)_{i=0}^{n-1}, a\right)=\left(g_{i+a}\right)_{i=0}^{n-1}, \quad \beta\left(\left(g_{i, j}\right)_{i, j=0}^{n-1, m-1},(b, c)\right)=\left(g_{i+b, j+c}\right)_{i, j=0}^{n-1, m-1}
$$

where all indexes are taken modulo $n$ and $n, m$ respectively. With respect to these actions semi-direct products $G \nmid \mathbb{Z}_{n}:=G^{n} \rtimes_{\alpha} \mathbb{Z}_{n}$ and $G \imath\left(\mathbb{Z}_{n} \times \mathbb{Z}_{m}\right):=G^{n m} \rtimes_{\beta}\left(\mathbb{Z}_{n} \times \mathbb{Z}_{m}\right)$ will be called wreath products of $G$ with $\mathbb{Z}_{n}$ and $G$ with $\left(\mathbb{Z}_{n} \times \mathbb{Z}_{m}\right)$ respectively. More general definition the reader can find in the book [25].

1.2. Structure of the paper. Section 2 is devoted to out main result - Theorem 2.5. Sections 3 and 4 include some preliminary facts about automorphisms of graphs of Morse functions on surfaces, and "combinatorial" structure of such functions on 2-torus. The proof of Theorem 2.5 is contained in Section 5.

\section{MAIN RESULT}

2.1. The class $\mathcal{P}$. First we recall the main result of [10]. For $n \in \mathbb{N}$, let $\mathcal{P}_{n}$ be a minimal set of isomorphism classes of groups which satisfies the following two conditions:

(1) the unit group $\{1\}$ belongs to $\mathcal{P}$,

(2) groups $A \times B$ and $A$ \Z $\mathbb{Z}_{n}$ belong to $\mathcal{P}$ whenever $A, B \in \mathcal{P}$, and $n \in \mathbb{N}$.

Let also $\mathcal{P}$ be a minimal set of isomorphism classes of groups which contains $\mathcal{P}_{n}$ as a set for each $n \in \mathbb{N}$. The following theorem gives the description of classes $\mathscr{G}(M), \mathscr{G}^{s m p}(M)$ and $\mathscr{G}^{g e n}(M)$ for all compact surfaces $M \neq S^{2}, T^{2}$.

Theorem 2.2 (Theorem $1.4[10]$ ). For each compact oriented surface $M \neq S^{2}, T^{2}$, $\mathscr{G}(M)=\mathcal{P}$, and $\mathscr{G}^{\mathrm{smp}}(M)=\mathcal{P}_{2}$. For all compact oriented surface $\mathscr{G}^{\text {gen }}(M)=\{1\}$.

2.3. Classes of groups $\mathcal{E}_{0}, \mathcal{E}_{1}$ and $\mathcal{E}_{2}$. To describe classes $\mathscr{G}\left(T^{2}\right)$ and $\mathscr{G}^{s m p}\left(T^{2}\right)$ we introduce three classes $\mathcal{E}_{0}, \mathcal{E}_{1}$ and $\mathcal{E}_{2}$ in the following way. Let $\mathcal{E}_{i}$ be a minimal set of isomorphism classes of groups such that

- $\mathcal{E}_{0}$ contains the group $A_{0} \imath\left(\mathbb{Z}_{n} \times \mathbb{Z}_{m n}\right), n, m \geq 1$ for each $A_{0} \in \mathcal{P}$,

- $\mathcal{E}_{1}$ contains the group $A_{1} \prec \mathbb{Z}_{n}, n \geq 1$ for each $A_{1} \in \mathcal{P}$,

- $\mathcal{E}_{2}$ contains the group $A_{2} \prec \mathbb{Z}_{n}, n \geq 1$ for each $A_{2} \in \mathcal{P}_{2}$.

Remark 2.4. Note that the group $\left(A \succ \mathbb{Z}_{2}\right) \times\left(B \nmid \mathbb{Z}_{3}\right)$ where $A, B \in \mathcal{P}$ belongs to $\mathcal{E}_{1}$ since it is isomorphic to the group $\left(\left(A<\mathbb{Z}_{2}\right) \times\left(B<\mathbb{Z}_{3}\right)\right)<\mathbb{Z}_{1}$. So classes $\mathcal{P}$ and $\mathcal{E}_{1}$ in general coincide, but we will consider the class $\mathcal{E}_{1}$ due to the convenience of a unique presentation of a group $G \in \mathcal{E}_{1}$ in the form $G=H \nmid \mathbb{Z}_{n}$ for some $n \geq 1$ and $H \in \mathcal{P}$.

It is well known that the Kronrod-Reeb graph of Morse functions on 2-torus is either a tree or contains a unique circuit, see Lemma 3.1 in [3]. The class of groups $G(f)$ for Morse functions $f$ on $T^{2}$ whose graphs are trees we will denote by $\mathscr{G}_{0}\left(T^{2}\right)$, otherwise, in the case of circuits, by $\mathscr{G}_{1}\left(T^{2}\right)^{1}$. The following theorem is our main result.

Theorem 2.5. The following classes coincide:

$$
\mathscr{G}_{0}\left(T^{2}\right)=\mathcal{E}_{0}, \quad \mathscr{G}_{1}\left(T^{2}\right)=\mathcal{E}_{1}, \quad \mathscr{G}^{g e n}\left(T^{2}\right)=\mathcal{E}_{2} .
$$

We prove Theorem 2.5 in Section 5. The proof is divided into three separate cases: for $\mathscr{G}_{0}\left(T^{2}\right), \mathscr{G}_{1}\left(T^{2}\right)$ and $\mathscr{G}^{\text {gen }}\left(T^{2}\right)$.

\footnotetext{
${ }^{1}$ Here indexes 0 and 1 correspond to the rank of $H_{1}\left(\Gamma_{f}, \mathbb{Z}\right)$
} 


\section{Automorphisms of GRAphs of FUnCtions on SURFACES}

In this section we want to show a precise way how the group $G(f)$ arises from the action of $\mathcal{S}^{\prime}(f)=\mathcal{S}(f) \cap \mathcal{D}_{\text {id }}(M)$ on $M$. Let $f: M \rightarrow \mathbb{R}$ be a Morse function on smooth oriented surface $M$, and $c$ be a real number. A connected component $C$ of the level-set $f^{-1}(c)$ is called critical, if $C$ contains at least one critical point of $f$, otherwise $C$ is called regular. Let $\Delta$ be a partition of $M$ into connected components of level-sets of $f$. It is well known that the quotient-space $\Gamma_{f}=M / \Delta$ has a structure of an 1-dimensional $\mathrm{CW}$ complex called a Kronrod-Reeb graph of $f$. For simplicity we will call it a graph of $f$. Let also $p_{f}: M \rightarrow \Gamma_{f}$ be a projection map. Then $f$ can be presented as the composition: $f=\widehat{f} \circ p_{f}: M \stackrel{p_{f}}{\longrightarrow} \Gamma_{f} \stackrel{\widehat{f}}{\longrightarrow} \mathbb{R}$. Denote by $\operatorname{Aut}\left(\Gamma_{f}\right)$ the group of homeomorphisms of the graph $\Gamma_{f}$. Note that each $h \in \mathcal{S}^{\prime}(f)$ preserves level-sets of $f$. Hence, $h \in \mathcal{S}^{\prime}(f)$ induces the homeomorphism $\rho(h)$ of $\Gamma_{f}$ such that the following diagram

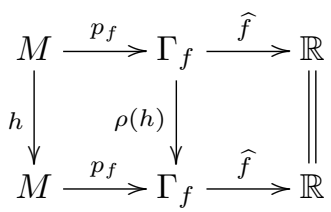

commutes, and the correspondence $h \mapsto \rho(h)$ is a homeomorphism $\rho: \mathcal{S}^{\prime}(f) \rightarrow \operatorname{Aut}\left(\Gamma_{f}\right)$. The image $\rho\left(\mathcal{S}^{\prime}(f)\right)$ is a finite group in $\operatorname{Aut}\left(\Gamma_{f}\right)$; we will denote it by $G(f)$.

\section{Combinatorial generalities on Morse functions on 2-torus And their GRAPHS}

We are interested in the "combinatorial" structure of Morse functions on $T^{2}$, so we will recollect some useful for us results on the structure of such functions. The following lemma holds.

Lemma 4.1 (Lemma 3.1 [3]). Let $f$ be a Morse function on $T^{2}$ and $\Gamma_{f}$ be its graph. Then $\Gamma_{f}$ is either a tree or contains a unique circuit.

We describe these two cases separately.

4.2. $\Gamma_{f}$ contains a circuit. Let $\Theta$ be a circuit in $\Gamma_{f}$. Let $C_{0} \subset T^{2}$ be a regular connected component of some level set $f^{-1}(c), c \in \mathbb{R}$, and $z$ be a point in $\Gamma_{f}$ corresponding to $C_{0}$. Obviously, $z$ belongs to the cycle $\Theta$ in $\Gamma_{f}$, iff $C_{0}$ does not separate $T^{2}$. Note that the level-set $f^{-1}(c)$ consists of a finite number of connected components, and is invariant under the action of any $h \in \mathcal{S}^{\prime}(f)$. Let $\mathcal{C}$ be the set $\left\{h\left(C_{0}\right) \mid h \in \mathcal{S}^{\prime}(f)\right\}$ of all images of $C_{0}$ under the action of elements from $\mathcal{S}^{\prime}(f)$. Then the set $\mathcal{C}$ consists of a finite number of components $\left\{C_{0}, C_{1}, \ldots, C_{n-1}\right\}$ of the set $f^{-1}(c)$ for some $n \geq 1$. Curves from $\mathcal{C}$ are pairwise disjoint, and since $C_{0}$ does not separate $T^{2}$, it follows that each $C_{i}$ also does not separate $T^{2}$. We can reorder the index set so that $C_{i}$ and $C_{i+1}$ bound a cylinder $Q_{i}$ such that the interior of $Q_{i}$ does not intersects with $\mathcal{C}$. Note that the group $\mathbb{Z}_{n}$ freely acts on the set of cylinders $\left\{Q_{i}\right\}$ by cyclic permutations. More about combinatorial description of Morse functions on 2-torus whose graphs contain circuits the reader can find in [23, 22].

4.3. $\Gamma_{f}$ is a tree. Let $f$ be a Morse function on $T^{2}$ with $\Gamma_{f}$ which is a tree. Then by [4, Theorem 2.5] there exists a unique vertex $v \in \Gamma_{f}$ such that each connected component of $T^{2}-p_{f}^{-1}(v)$ is an open 2-disk. Such vertex $v$ of $\Gamma_{f}$ and the connected component $V=p_{f}^{-1}(v)$ of the corresponding to $v$ critical level-set of $f$ will be called special. Note that the topological structure of the atom of $V$, i.e., a regular neighborhood of $V$ which consists of connected components of level-set of $f$ and does not contain other critical points of $f$, is well understood [2]. 
Next we describe a special subgroup of $G(f)$ which plays an important role in an algebraic description of $G(f)$. The group $G(f)$ acts on the graph $\Gamma_{f}$, and we let $G_{v}$ be a stabilizer of $v$ with respect to this $G$-action on $\Gamma_{f}$. The set $G_{v}^{l o c}=\left\{\left.g\right|_{\text {st }(v)} \mid g \in G_{v}\right\}$ which contains restrictions of elements of $G_{v}$ onto the star st $(v)$ is a subgroup of $\operatorname{Aut}(\operatorname{st}(v))$, we will call it a local stabilizer of $v$. It is well known that the group $G_{v}^{\text {loc }}$ is isomorphic to the product $\mathbb{Z}_{n} \times \mathbb{Z}_{m n}$ for some $n, m \geq 1$, see [4, Theorem 2.5]. More information the reader can find in $[2,16]$.

4.4. Algebraic structure of $G(f)$. It turns out that the combinatorial information from the subsections 4.2 and 4.3 is enough to describe an algebraic structure of the groups $G(f)$ for Morse functions on $T^{2}$.

Lemma 4.5 (Theorem $3.2[3]$ ). Let $f$ be a Morse function on $T^{2}$, and $\Gamma_{f}$ be its graph .

(1) If $\Gamma_{f}$ contains a unique circle, then the group $\mathbb{Z}_{n}$ acts on the set of cylinders $\left\{Q_{i}\right\}_{i=0}^{n-1}$ by cyclic permutations and there is an isomorphism $G(f)=G\left(\left.f\right|_{Q_{0}}\right) \mathbb{Z}_{n}$, where $n$ is a cyclic index of $f$, and $Q_{0}$ is a cylinder bounded by parallel curves $C_{0}$ and $C_{1}$.

(2) If $\Gamma_{f}$ is a tree, then there exists a special vertex $v$ in $\Gamma_{f}$. Let also $V=p_{f}^{-1}(v)$ be a special component of critical level set of $f$ which corresponds to $v$ and $N$ be an atom of $V$. Moreover the group $G_{v}^{\text {loc }}$ freely acts by diffeomorphisms from $\mathcal{S}^{\prime}(f)$ on $T^{2}$, this action induces a free action of $G_{v}^{\text {loc }}$ on connected components of $\overline{T^{2}-N}$, and so there exists a set of 2 -disks $\left\{D_{0}, D_{1}, \ldots, D_{r}\right\}$ such that $G(f)=$ $\prod_{i=0}^{r} G\left(\left.f\right|_{D_{i}}\right)$ 乙 $G_{v}^{\text {loc }}$, where $r$ is a number of orbits of free $G$-action on $T^{2}$.

4.6. Morse equality. We would like to recall the relationship between Euler characteristic of the surface and Morse function defined on it. This connection is given by the outstanding Morse equality - it is an important ingredient needed for the proof of Theorem 2.5.

Theorem 4.7 (Morse equality). Let $f$ be a Morse function on smooth compact and oriented surface $M$ without boundary and let $c_{i}(f)$ be a number of critical points of $f$ of index $i, i=0,1,2$. Then the following equality holds

$$
\chi(M)=c_{0}(f)-c_{1}(f)+c_{2}(f) .
$$

It is well known that $\chi\left(T^{2}\right)=0$, so Morse equality for a Morse function $f$ on $T^{2}$ has the form: $c_{0}(f)+c_{2}(f)=c_{1}(f)$.

\section{Proof of TheOrem 2.5}

Obviously the inclusion $\mathscr{G}_{i}\left(T^{2}\right) \hookrightarrow \mathcal{E}_{i}$ for $i=0,1$ directly follows from Lemma 4.5 because of the structure of the class $\mathcal{E}_{i}$. So to prove Theorem 2.5 we have to establish the reverse inclusion $\mathcal{E}_{i} \hookrightarrow \mathscr{G}_{i}\left(T^{2}\right)$ for $i=0,1$. In other words we need to prove that for each $A \in \mathcal{E}_{i}$ there exists a Morse function $f$ on $T^{2}$ such that $A \cong G(f)$. These will be done below in Subsections 5.1 and 5.2. The case of simple Morse functions will be considered in Subsection 5.3.

5.1. Case 1: Functions, whose graphs have circuits. Let $A$ be a group from $\mathcal{E}_{1}$. So the group $A$ has the form $B \nmid \mathbb{Z}_{n}$ for some $B \in \mathcal{P}$ and some $n \geq 1$. We divide the proof of the inclusion $\mathcal{E}_{1} \hookrightarrow \mathscr{G}_{1}\left(T^{2}\right)$ into two steps. First we have to define a Morse function $f_{0}: T^{2} \rightarrow \mathbb{R}$ such that $\Gamma_{f_{0}}$ contains a circuit and $G\left(f_{0}\right) \cong \mathbb{Z}_{n}$ for $n$ as above. Then for the given group $B$ we change $f_{0}$ on a neighborhood of maximums of $f_{0}$ to obtain another Morse function $f: T^{2} \rightarrow \mathbb{R}$ such that $G(f) \cong B \gtrless \mathbb{Z}_{n}$. 
One can define such a Morse function $f_{0}$ by the following procedure. Let $Q_{i}=$ $S^{1} \times[0,1], i=0, \ldots, n-1$ be a cylinder and $g_{i}: Q_{i} \rightarrow \mathbb{R}$ be a Morse function such that $g_{i}$ has one maximum $l_{i}$, one minimum $p_{i}$, two saddles $s_{i 1}, s_{i 2}$ and

$$
g\left(\partial Q_{i}\right)=0, \quad g\left(l_{i}\right)=1, \quad g\left(p_{i}\right)=-1, \quad g\left(s_{i j}\right)= \pm 1 / 2, \quad j=1,2 .
$$

Consider the same orientation on each $Q_{i}$; it canonically induces the orientations of the connected components of the boundary $\partial Q_{i}=\partial Q_{i}^{0} \cup \partial Q_{i}^{1}$, where $\partial Q_{i}^{j}=S^{1} \times\{j\}$, $j=0,1$.

If $n=1$ attach the boundaries of $Q_{0}$ by identity diffeomorphism. The resulting surface is a 2-torus. Since the values of $g$ on $\partial Q_{0}^{0}$ and $\partial Q_{0}^{1}$ coincide, it follows that $g_{0}$ induces a unique Morse function $f_{0}$ on $T^{2}$. If $n \geq 1$ attach all $Q_{i}$ together in cyclic order by identity diffeomorphism of $\partial Q_{i}^{1} \rightarrow \partial Q_{i+1}^{0}$ where the index $i$ takes modulo $n$. The resulting surface is obviously a 2 -torus, and since the values $g_{i}$ on connected components of the boundary of $Q_{i}$ coincide, it follows that $g_{0}$ induces a unique smooth function $f_{0}$ on $T^{2}$ such that $\Gamma_{f_{0}}$ contains a circuit and $G\left(f_{0}\right) \cong \mathbb{Z}_{n}$. Points $l_{i}, p_{i}$ and $s_{i j}$ are the corresponding maximums, minimums and saddle points of $f_{0}, i=0, \ldots, n-1, j=1,2$.

Let $D_{i}$ be a neighborhood of the maximum $l_{i}$ of $f_{0}$ which does not contain other critical points and consists of connected components of level-sets of $f$. For the given group $B$ by Theorem 2.2 there exists a smooth function $f_{i}$ on a 2-disk $D_{i}$ such that $G\left(f_{i}\right) \cong B$. Next we change the function $f_{0}$ on $D_{i}$ by replacing $\left.f_{0}\right|_{D_{i}}$ to $f_{i}$ on $D_{i}$; the resulting function we denote by $f$. By Lemma $4.5, f$ is such that $\Gamma_{f}$ contains a unique circuit and $G(f) \cong B \imath \mathbb{Z}_{n}$. So we proved that the inclusion $\mathcal{E}_{1} \hookrightarrow \mathscr{G}_{1}\left(T^{2}\right)$ holds.

5.2. Case 2: Functions, whose graphs are trees. Let $A$ be a group from $\mathcal{E}_{0}$. We need to show that there exists a Morse functions $f$ on $T^{2}$ such that $\Gamma_{f}$ is a tree and $G(f) \cong A$. From the definition of the class $\mathcal{E}_{0}$ there exist $n, m \geq 1$ and $B \in \mathcal{P}$ such that $A=B$ ? $\left(\mathbb{Z}_{n} \times \mathbb{Z}_{m n}\right)$. As in the Case 1 we divide our proof into two steps. First for a given $n, m \geq 1$ we define a Morse function $f_{0}: T^{2} \rightarrow \mathbb{R}$ such that $\Gamma_{f_{0}}$ is a tree and $G\left(f_{0}\right)=\mathbb{Z}_{n} \times \mathbb{Z}_{m n}$, and finally for the given group $B$ we change the function $f_{0}$ to obtain the Morse function $f: T^{2} \rightarrow \mathbb{R}$ such that $G(f) \cong B$ ? $\left(\mathbb{Z}_{n} \times \mathbb{Z}_{m n}\right)$.

To do the first step we need some preliminaries. Let $\gamma: \mathbb{R}^{2} \times \mathbb{Z}^{2} \rightarrow \mathbb{R}^{2}$ be a free action of $\mathbb{Z}^{2}$ given by the formula $\gamma((x, y),(a, b))=(x+2 a, y+2 b)$. For $n, m \geq 1$ as above this action induces a free action $\delta$ of the subgroup $n \mathbb{Z} \times m n \mathbb{Z}$ of $\mathbb{Z}^{2}$ on $\mathbb{R}^{2}$ by the formula $\delta((x, y),(a, b))=(x+2 n a, y+2 m n b)$. Note that the rectangle $P=[0,2 n] \times[0,2 m n]$ is a fundamental domain for the action $\delta$, and the quotient space $\mathbb{R}^{2} / \delta$ is a 2-torus. Let $D_{k l}$ be a square $[k, k+1] \times[l, l+1] \subset \mathbb{R}^{2}, k, l \in \mathbb{Z}$, and $q: \mathbb{R}^{2} \rightarrow \mathbb{R}^{2} / \delta$ be the projection map, and $\mathrm{B}$ be the set of images of $D_{k l}$ with respect to $q$. The action $\delta$ induces a free action $\sigma$ of $\mathbb{Z}_{n} \times \mathbb{Z}_{m n}$ on the set $\mathrm{B}$. The number of orbits of this action is equal to

$$
\frac{\#\left(\text { squares } D_{k l} \text { belonging to } P\right)}{\#\left(\mathbb{Z}_{n} \times \mathbb{Z}_{m n}\right)}=\frac{4 n^{2} m}{n^{2} m}=4 \text {. }
$$

So all disks $D_{k l}=[k, k+1] \times[l, l+1]$ in $P$ can be enumerated as $D_{\text {rij }}$ by three indexes $r=1,2,3,4, i=0,1, \ldots, n-1$, and $j=0,1, \ldots, m n-1$. Let $m_{r i j}$ be an internal point of $D_{\text {rij }}$.

Then there exists a double-periodic Morse function $g_{0}: \mathbb{R}^{2} \rightarrow \mathbb{R}$ such that $g_{0} \circ \delta_{a, b}=g_{0}$ for all $(a, b) \in \mathbb{Z}^{2}$, where $\delta_{a, b}(x, y)=\delta((x, y),(a, b))$, and on $P$ it satisfies:

- $g_{0}(\{k, l\})=0$ is a saddle point, $k=0,1, \ldots, 2 n-1, l=0,1, \ldots, 2 m n-1$,

- $g_{0}$ has a unique maximum at $m_{r 00}$ for $r=1,2$, and a unique minimum for $r=3,4$ and such that

$g_{0}\left(m_{100}\right)=1, \quad g_{0}\left(m_{200}\right)=2, \quad g_{0}\left(m_{300}\right)=-1, \quad g_{0}\left(m_{400}\right)=-2$. 
It is easy to see that $g_{0}$ induces a unique Morse function on $T^{2}=\mathbb{R}^{2} / \delta$, which we denote by $f_{0}$. The resulting function $f_{0}$ is such that $\Gamma_{f_{0}}$ is a tree, and $G\left(f_{0}\right)=\mathbb{Z}_{n} \times \mathbb{Z}_{m n}$.

It remains to modify $f_{0}$ using the group $B$ as above. Since the action $\sigma$ on B has 4 orbits, choose one of them, say when $r=1$. By Theorem 2.2 there exists a Morse function $f_{1 i j}$ on $D_{1 i j}$ such that $G\left(f_{1 i j}\right) \cong B$ for all $i=0, \ldots, n-1$ and $j=0, \ldots, m n-1$. We change the function $f_{0}$ on $D_{1 i j}$ by replacing of $\left.f_{0}\right|_{D_{1 i j}}$ to $f_{1 i j}$, and the resulting function we will denote by $f$. Note that $G\left(\left.f\right|_{D_{r i j}}\right)=1$ for $r=2,3,4$ and $i=0,1, \ldots, n-1$, $j=0,1, \ldots, n m-1$ by definition of $f_{0}$. Then by Lemma 4.5

$$
\begin{aligned}
G(f) & \cong \prod_{r=1}^{4} G\left(\left.f\right|_{D_{r 00}}\right) \curlywedge\left(\mathbb{Z}_{n} \times \mathbb{Z}_{m n}\right) \\
& =G\left(\left.f\right|_{D_{100}}\right) \curlywedge\left(\mathbb{Z}_{n} \times \mathbb{Z}_{m n}\right) \\
& \cong B \curlywedge\left(\mathbb{Z}_{n} \times \mathbb{Z}_{m n}\right) .
\end{aligned}
$$

So we proved that the inclusion $\mathcal{E}_{0} \hookrightarrow \mathscr{G}_{0}\left(T^{2}\right)$ holds.

5.3. Case 3: Simple Morse functions. It is easy to see that there exist simple Morse functions on $T^{2}$ in the case when its graph contains a unique circuit. Indeed, a Morse function $f$ on $T^{2}$ such that $\Gamma_{f}$ contains a unique circuit is simple if the restriction $\left.f\right|_{Q_{i}}$ is simple for each $i=0,1, \ldots, n$. The following lemma shows that this is the only case.

Lemma 5.4. Let $f$ be a simple Morse function on $T^{2}$. Then $\Gamma_{f}$ is not a tree.

Proof. Assume that $f$ is simple and $\Gamma_{f}$ is a tree. Then there exists a unique special vertex $v$ of the tree $\Gamma_{f}$. Let $V=p_{f}^{-1}(v)$ be a special component of critical level-set of $f$ which corresponds to $v$. Note that the special component $V$ contains "many" saddles of $f$. Let also $N$ be a regular neighborhood of $V$ which consists of level-sets of $f$ and does not contain other critical points. Since $v$ is a special vertex, it follows that $\overline{T^{2}-N}$ is a disjoint union of 2-disks, say $\left\{D_{i}\right\}_{i=1}^{n}$ for some $n \in \mathbb{N}$. Note that the restriction $\left.f\right|_{D_{i}}$ for all $i=0,1, \ldots, n$ is also a simple Morse function since $f$ is simple.

Next we change the function $f$ in the following way: replace $\left.f\right|_{D_{i}}$ by the function on 2-disk $D_{i}$ which has the only one critical point, $i=1, \ldots, n$; the resulting function we denote by $g$. After these changes the function $g$ satisfies the following conditions:

(1) $\Gamma_{g}$ is also a tree, since all changes of $f$ were on the connected components of the complement of $N$ being 2-disks,

(2) $V$ is also a critical level-set of $g$, but all saddles of $g$ belong to $V$,

(3) $g$ is simple since $f$ was simple.

Since $g$ is simple (by assumption on $f$ and by construction), it follows that the number of saddles belonging to $V$ must be equal to 1 , and so $c_{1}(g)=1$. From Morse equality (Theorem 4.7) we have $c_{1}(g)=c_{0}(g)+c_{2}(g)$, and so $1=c_{0}(g)+c_{2}(g)$. This is an inconsistency. Hence if $f$ is simple, then $\Gamma_{f}$ is not a tree.

Now we need to show that the classes $\mathscr{G}^{s m p}\left(T^{2}\right)$ and $\mathcal{E}_{2}$ coincide. First we show the inclusion $\mathscr{G}^{s m p}\left(T^{2}\right) \hookrightarrow \mathcal{E}_{2}$ holds. Let $f$ be a simple Morse function on $T^{2}$. By Lemma 5.4 the graph $\Gamma_{f}$ has a unique circle. The restriction $\left.f\right|_{Q_{0}}$ is simple Morse function on cylinder $Q_{0}$ so $G\left(\left.f\right|_{Q_{0}}\right)$ belongs to the class $\mathcal{P}_{2}$. By (1) Lemma 4.5 the group $G(f)$ is isomorphic to $G\left(\left.f\right|_{Q_{0}}\right) \gtrless \mathbb{Z}_{n}$ for some $n \geq 1$ which depends on the function $f$, and so $G(f)$ belongs to $\mathcal{E}_{2}$ by definition of the class $\mathcal{E}_{2}$.

The reverse inclusion $\mathcal{E}_{2} \hookrightarrow \mathscr{G}^{s m p}\left(T^{2}\right)$ follows from the procedure defined in Case 1 with $B \in \mathcal{P}_{2}$ as sub-case. Theorem is proved.

Acknowledgments. Authors would like to express their gratitude to Sergiy Maksymenko for advises and discussions. 


\section{REFERENCES}

1. Kree Cole-McLaughlin, Herbert Edelsbrunner, John Harer, Vijay Natarajan, and Valerio Pascucci, Loops in Reeb graphs of 2-manifolds, Discrete Comput. Geom. 32 (2004), no. 2, 231-244.

2. Bohdan Feshchenko, Actions of finite groups and smooth functions on surfaces, Methods Funct. Anal. Topology 22 (2016), no. 3, 210-219.

3. Bohdan Feshchenko, Deformations of functions on 2-torus; arXiv:1903.01753, 2019.

4. B. Feshhenko, Deformations of smooth functions on 2-torus, whose kr-graph is a tree, Proceedings of Institute of Mathematics of NAS of Ukraine 12 (2015), no. 6, 22-40.

5. Kazuichi Ikegami and Osamu Saeki, Cobordism group of Morse functions on surfaces, J. Math Soc. Japan 55 (2003), no. 4, 1081-1094.

6. Boldizsár Kalmár, Cobordism group of Morse functions on unoriented surfaces, Kyushu J. Math. 59 (2005), no. 2, 351-363.

7. Marek Kaluba, Wacław Marzantowicz, and Nelson Silva, On representation of the Reeb graph as a sub-complex of manifold, Topol. Methods Nonlinear Anal. 45 (2015), no. 1, 287-307.

8. Anna Kravchenko and Sergiy Maksymenko, Automorphisms of Kronrod-Reeb graphs of Morse functions on 2-sphere, Proceedings of the International Geometry Center 11 (2018), no. 4, $72-79$.

9. Anna Kravchenko and Sergiy Maksymenko, Automorphisms of cellular divisions of 2-sphere induced by functions with isolated critical points; arXiv:1911.10808, 2019.

10. Anna Kravchenko and Sergiy Maksymenko, Automorphisms of Kronrod-Reeb graphs of Morse functions on compact surfaces, European Journal of Mathematics, 2020, 18 pages; arXiv:1808.08746 doi: 10.1007/s40879-019-00379-8.

11. E. A. Kudryavtseva, Realization of smooth functions on surfaces as height functions, Mat. Sb. 190 (1999), no. 3, 29-88.

12. E. A. Kudryavtseva, The topology of spaces of Morse functions on surfaces, Math. Notes 92 (2012), no. 1-2, 219-236. Translation of Mat. Zametki 92 (2012), no. 2, 241-261.

13. E. A. Kudryavtseva, On the homotopy type of spaces of Morse functions on surfaces, Mat. Sb. 204 (2013), no. 1, 79-118.

14. E. V. Kulinich, On topologically equivalent Morse functions on surfaces, Methods Funct. Anal. Topology 4 (1998), no. 1, 59-64.

15. D. P. Lychak and A. O. Prishlyak, Morse functions and flows on nonorientable surface, Methods Funct. Anal. Topology 15 (2009), no. 3, 251-258.

16. S. Maksymenko and B. Feshchenko, Homotopy properties of spaces of smooth functions on 2-torus, Ukrainian Math. J. 66 (2014), no. 9, 1205-1212.

17. Sergiy Maksymenko, Homotopy types of stabilizers and orbits of Morse functions on surfaces, Ann. Global Anal. Geom. 29 (2006), no, 3, 241-285.

18. Sergiy Maksymenko, Functions on surfaces and incompressible subsurfaces, Methods Funct. Anal. Topology 16 (2010), no. 2, 167-182.

19. Sergiy Maksymenko, Functions with isolated singularities on surfaces, Geometry and topology of functions on manifolds. Pr. Inst. Mat. Nats. Akad. Nauk Ukr. Mat. Zastos. 7 (2010), no. 4 , $7-66$.

20. Sergiy Maksymenko, Homotopy types of right stabilizers and orbits of smooth functions on surfaces, Ukrainian Math. J. 64 (2012), no. 9, 1186-1203.

21. Sergiy Maksymenko, Deformations of functions on surfaces by isotopic to the identity diffeomorphisms, 45 pages; arXiv:math/1311.3347, 2014.

22. Sergiy Maksymenko and Bohdan Feshchenko, Functions on 2-torus whose Kronrod-Reeb graph contains a cycle, Methods Funct. Anal. Topology 21 (2015), no. 1, 22-40.

23. Sergiy Maksymenko and Bohdan Feshchenko, Orbits of smooth functions on 2-torus and their homotopy types, Matematychni Studii 44 (2015), no. 1, 67-83.

24. Yasutaka Masumoto and Osamu Saeki, A smooth function on a manifold with given Reeb graph, Kyushu J. Math. 65 (2011), no. 1, 75-84.

25. J. D. P. Meldrum, Wreath products of groups and semigroups, vol. 74 of Pitman Monographs and Surveys in Pure and Applied Mathematics, Longman, Harlow, 1995.

26. Lukasz Patryk Michalak, Realization of a graph as the Reeb graph of a Morse function on a manifold, Topol. Methods Nonlinear Anal. 52 (2018), no. 2, 749-762.

27. E. A. Polulyakh, Kronrod-Reeb graphs of functions on noncompact two-dimensional surfaces. I, Ukrainian Math. J. 67 (2015), no. 3, 431-454; Translation of Ukrain. Mat. Zh. 67 (2015), no. $3,375-396$.

28. E. A. Polulyakh, Kronrod-Reeb graphs of functions on noncompact two-dimensional surfaces. II, Ukrainian Math. J. 67 (2016), no. 10, 1572-1583. Translation of Ukrain. Mat. Zh. 67 (2015), no. $10,1398-1408$. 
29. V. V. Sharko, Functions on surfaces. I, In Some problems in contemporary mathematics (Russian), vol. 25 of Pr. Inst. Mat. Nats. Akad. Nauk Ukr. Mat. Zastos., pp. 408-434, Kiev, Inst. Mat. Nats. Akad. Nauk Ukr., 1998.

30. V. V. Sharko, Smooth and topological equivalence of functions on surfaces, Ukrain. Mat. Zh. 55 (2003), no. 5, 687-700.

31. V. V. Sharko, About Kronrod-Reeb graph of a function on a manifold, Methods Funct. Anal. Topology 12 (2006), no. 4, 389-396.

Taras Shevchenko National University of Kyiv, 60 Volodymyrska str., Kyiv, 01033, Ukraine E-mail address: annakravchenko1606@gmail.com

Institute of Mathematics, National Academy of Sciences of Ukraine, 3 Tereshchenkivs'ka STR., KYIV, 01601, UkRaine

E-mail address: fb@imath.kiev.ua

Received 11/12/2019; Revised 31/01/2020 